\section{ATRIBUTOS DE CALIDAD Y VIDA DE ANAQUEL DE FRUTOS DE CULTIVARES DE CHILE SERRANO EN MÉXICO}

\section{FRUIT QUALITY ATTRIBUTES AND SHELF LIFE OF SERRANO PEPPER CULTIVARS IN MÉXICO}

\author{
Enrique Vázquez García ${ }^{1 *}$, Moisés Ramírez \\ Meraz $^{1}$, Horacio Mata Vázquez ${ }^{1}$, Rafael Ariza \\ Flores $^{2}$ e Irán Alia Tejacal ${ }^{3}$
}

\begin{abstract}
${ }^{1}$ Campo Experimental Las Huastecas, Centro de Investigación Regional del Noreste, Instituto Nacional de Investigaciones Forestales, Agrícolas y Pecuarias (INIFAP). Km 55 carretera Tampico-Mante. México C.P. 89610. Tel y Fax: 836 2760168. ${ }^{2}$ Campo Experimental Chilpancingo, Centro de Investigación Regional Golfo-Centro. INIFAP, Av. Rufo Figueroa s/n, Col. Burócratas. 39090, Chilpancingo, Guerrero, México. ${ }^{3}$ Facultad de Ciencias Agropecuarias, Universidad Autónoma del Estado de Morelos. Av. Universidad 1001. 62210, Cuernavaca, Morelos, México.
\end{abstract}

* Autor para correspondencia (vazquez.enrique@inifap.gob.mx)

\section{RESUMEN}

El chile Serrano (Capsicum annuum L.) se consume en México básicamente en fresco, por lo que la calidad y la vida de anaquel de sus frutos son factores importantes en su comercialización; sin embargo, la literatura científica sobre esta temática es escasa. En este estudio se cuantificaron los atributos de calidad y vida de anaquel de 19 cultivares de chile Serrano desarrollados por el Instituto Nacional de Investigaciones Forestales, Agrícolas y Pecuarias (INIFAP) y por varias compañías particulares de semillas. Se utilizó un diseño experimental completamente al azar con 10 repeticiones, y cada fruto se consideró como una repetición. Se encontraron diferencias entre cultivares en peso de fruto, diámetro, longitud, firmeza, color (croma y tono o hue) y pérdida fisiológica de peso. Los cultivares 'Bandido' y 'HMX-5651' superaron ( $\mathrm{P} \leq \mathbf{0 . 0 1})$ a los demás en peso e intensidad de color. Los materiales 'HMX-6671', 'HMX-6661', 'Blakie' y 'HS-44' destacaron por su intensidad de color. 'Centauro' fue el material con mayor valor de firmeza ( $P \leq$ 0.01). Los materiales 'HS-49', 'HS-51', '21-20-1' y '74-5-5' registraron altos valores de firmeza y bajos porcentajes de pérdida de peso. Los cultivares '74-27-5', 'Tampiqueño 74' y '33-12-2' presentaron valores bajos de pérdida de peso. Los materiales desarrollados por las compañías particulares sobresalieron por su mayor peso e intensidad de color, mientras que los cultivares desarrollados por el INIFAP destacaron por su mayor firmeza y menor pérdida de peso.

Palabras claves: Capsicum annuum, firmeza, pérdida de peso, croma, tono.

Recibido: 09 de Febrero del 2010.

Aceptado: 03 de Junio del 2010.

\section{SUMMARY}

Serrano pepper (Capsicum annuum L.) is mostly used in México for fresh consumption. Thus, fruit quality and fruit shelf life are important factors for its marketing. However, scientific literature on this subject is scarce. In this study we evaluated fruit quality attributes and shelf life of $\mathbf{1 9}$ cultivars of Serrano pepper developed by the Instituto Nacional de Investigaciones Agrícolas, Forestales y Pecuarias (INIFAP), and several private seed companies. A completely random experimental design was used with 10 repetitions, each fruit being a repetition. Cultivars varied $(P \leq 0.01)$ in: fruit weight, diameter, length, firmness, color (chroma and tone or hue angle) and physiological weight loss. Fruits from 'Bandido' and 'HMX-5651' cultivars were the largest and had the highest color intensity (P $\leq$ 0.01), while 'HMX-6671' 'HMX-6661', 'Blakie', 'HS44' produced fruits of intense color $(P \leq 0.01)$. 'Centauro' fruits had the highest firmness (P $\leq$ 0.01). Cultivars 'HS-49', 'HS-51', '21-20-1' and '74-5-5' registered high values of firmness combined with low percentage of weight loss. Cultivars '74-27-5', 'Tampiqueño 74' and '33-12-2' also had low weight losses. Materials developed by private companies produced large fruits of intense color, while cultivars developed by INIFAP had higher fruit firmness and lower weight loss.

Index words: Capsicum annuum, firmness, weight loss, chroma, tone.

\section{INTRODUCCIÓN}

El cultivo del chile (Capsicum spp.) es importante en la historia, tradición y cultura de México y es, además, un producto agrícola con alta demanda mundial, ya que se ubica entre las siete hortalizas más cultivadas del mundo, con una producción mundial estimada de 24 millones de toneladas (Pérez-Castañeda et al., 2008).

México cuenta con la mayor variabilidad genética de Capsicum annuum var. annuиm y de sus parientes silvestres $C$. annuиm var. aviculare y $C$. annuиm var. glabriusculum, representada por numerosos tipos (Serranos, Jalapeños, Pasillas, Guajillos, de Árbol, y otros.) adaptados a diferentes condiciones agroecológicas y ampliamente usados en el país. Asimismo, cuenta con otras especies importantes de chile como $C$. chinense y $C$. pubescens, mejor conocidos como chiles Habanero y Manzano, respectivamente (Laborde y Pozo, 1982; Meneses et al., 2006; Hernández et al., 2008).

El chile Serrano es uno de los más comerciales en el país donde goza de preferencia para su consumo en fresco, por lo que la calidad del fruto y su vida de anaquel son factores importantes en su valor comercial. Existe abundante información sobre los factores que afectan la calidad y vida de anaquel de diversos tipos de chile, en especial del tipo Pimiento morrón; sin embargo, la literatura científica sobre esta temática es escasa para el chile Serrano. Diversos autores coinciden en que los principales factores que demeritan la calidad y vida de anaquel de los diversos tipos de chiles son la pérdida 
fisiológica de peso y los cambios de color del epicarpio (Lownds et al., 1994; Martínez et al., 2005; Díaz-Pérez et al., 2006; Smith et al., 2006). En este contexto la presente investigación tuvo como objetivo cuantificar los atributos de calidad y vida de anaquel de 19 variedades mejoradas de chile Serrano, desarrolladas por el Instituto Nacional de Investigaciones Forestales Agrícolas y Pecuarias (INIFAP) y por varias compañías particulares productoras de semillas.

\section{MATERIALES Y MÉTODOS}

El trabajo se llevó a cabo en Tamaulipas, con plantas crecidas en campo a $22^{\circ} 34^{\prime} \mathrm{LN}, 98^{\circ}$ 09' LO. En diciembre de 2007 se cosechó un ensayo de rendimiento del programa de mejoramiento genético de chile del INIFAP, integrado por 19 variedades de chile Serrano con las siguientes características: seis líneas avanzadas F8 del INIFAP con un grado de endogamia cercano a $99 \%$ ('HS-49', 'HS-51', '74-27-5', '21-20-1', '74-5-5' y '3312-2'); cuatro variedades comerciales liberadas por el mismo Instituto entre 1974 y 2007 ('Coloso', 'HS-44', 'Centauro' y 'Tampiqueño 74'; Pozo, 1981; Ramírez et al., 2007); y nueve variedades desarrolladas por compañías particulares ('HMX-5651', 'HMX-6661', 'HMX-6671' y 'Bandido', de Harris Moran; 'Lucero', de Mar Seed; 'Milenio', de Caloro; 'Blakie', 'Nazas' y 'Tuxtlas', de Seminis). Inmediato a la cosecha, los frutos se trasladaron al laboratorio de postcosecha donde visualmente se eligieron 20 ejemplares de cada cultivar, en los que se hizo la evaluación, en la etapa de madurez fisiológica de color verde uniforme.

A 10 de los 20 frutos seleccionados de cada variedad se les determinó peso $(\mathrm{g})$, diámetro $(\mathrm{cm})$, longitud $(\mathrm{cm})$, firmeza $\left(\mathrm{N} \mathrm{cm}^{-2}\right)$, porcentaje de llenado de placenta y grosor de pericarpio $(\mathrm{mm})$, mediciones que se hicieron el mismo día de la cosecha (Martínez et al., 2003). A los 10 frutos restantes de cada variedad se les determinó el color del epicarpio (croma y tono o hue) y el porcentaje de pérdida fisiológica de peso (Martínez et al., 2003), medidos a los $12 \mathrm{~d}$ posteriores a la cosecha. La firmeza se midió con un penetrómetro de frutas Wagner ${ }^{\circledR}$, provisto con un puntal de $0.5 \mathrm{~cm}$; esta medición se basó en la presión $\left(\mathrm{N} \mathrm{cm}^{2}\right)$ necesaria para penetrar el pericarpio del fruto. El color se midió con un colorímetro Minolta modelo $410 \AA$, el cual proporcionó directamente los valores de croma y tono. La vida de anaquel de los frutos se evaluó mediante la pérdida fisiológica de peso y los cambios de color del epicarpio. Se consideró que a mayor pérdida de peso y mayores cambios en los valores de croma y tono en función del tiempo, el fruto tendría menor vida de anaquel, como lo han demostrado varios autores (Lownds et al., 1994; Martínez et al., 2005;
Díaz-Pérez et al., 2006; Smith et al., 2006). El manejo técnico del ensayo de rendimiento en campo se hizo de acuerdo con el paquete tecnológico recomendado por el INIFAP para este cultivo (INIFAP, 1999).

El peso de los frutos se midió con una báscula digital Torrey modelo EQ 5/10® y el diámetro y longitud se midieron con un vernier Surtek® ${ }^{\circledR}$. Se utilizó un diseño experimental completamente al azar con 10 repeticiones, y cada fruto se consideró como una unidad experimental. Con la información recabada se hicieron análisis de varianza, pruebas de comparación de medias $(\mathrm{P} \leq 0.01)$ y análisis de correlación de Pearson, todos con el programa estadístico SAS versión 6.01 (SAS Institute, 1998).

\section{RESULTADOS Y DISCUSIÓN}

Se encontraron diferencias entre variedades en peso y diámetro del fruto $(\mathrm{P} \leq 0.01)$, donde destacaron por su mayor peso las variedades 'Bandido' y 'HMX-5651' (12.2 y $11.8 \mathrm{~g} /$ fruto). En general, las variedades del INIFAP registraron un tamaño intermedio, y dentro de este grupo sobresalieron 'HS-51' y 'HS-49' (10.5 y 10.1 $\mathrm{g} /$ fruto). Se demostró que hubo una correlación positiva entre peso y diámetro de fruto $(r=0.70, \alpha=0.01)$, coincidente con lo reportado por Díaz-Pérez et al. (2006). Las variedades 'HMX-6671', 'HMX-6661', 'Lucero', '74-27-5' y 'Blakie' produjeron frutos más largos (P $\leq$ 0.01) que el resto de ellas (Cuadro 1).

Al hacer cruzas entre siete progenitores, Martínez et al. (2005) consignaron pesos individuales de frutos de chile Serrano entre 5.5 y $15 \mathrm{~g} /$ fruto, con una media de 9.6 $\mathrm{g} /$ fruto. Según estos autores, las diferencias entre progenitores y cruzas para peso de fruto se explicaron solamente por efectos genéticos no aditivos, por lo que ellos sugieren que las mejores estrategias de mejoramiento serían la formación de híbridos, y con el uso de métodos de selección recurrente que exploten los efectos no aditivos en caracteres determinados por efecto de dominancia, como peso individual del fruto y rendimiento por hectárea.

En firmeza de fruto, cinco de las variedades del INIFAP ('Centauro', 'HS-49', 'HS-51', '21-20-1' y '74$\left.5-5^{\prime}\right)$ se ubicaron entre las de mayor valor $(\mathrm{P} \leq 0.01)$ con respecto a las demás (Cuadro 1), en especial 'Centauro' (159 $\left.\mathrm{N} \mathrm{cm}^{-2}\right)$; por el contrario, siete variedades provenientes de compañías particulares registraron los valores más bajos de firmeza (98 a $128 \mathrm{~N} \mathrm{~cm}^{-2}$ ). En porcentaje de llenado de placenta y grosor de pericarpio no hubo diferencias varietales $(\mathrm{P}>0.01)$. 
En cuanto al color del fruto determinado al momento de la cosecha, ocho de las 19 variedades presentaron un intenso color verde, superior al resto de materiales $(\mathrm{P} \leq$ $0.01)$, de los cuales siete fueron de compañías particulares ('Bandido', 'HMX-5651', 'HMX-6671', 'HMX-6661', 'Blakie', 'Nazas' y 'Tuxtlas') y uno de INIFAP ('HS44'); ese color correspondió con valores bajos en croma (9 a 17) y altos en tono (129 a 134). Estas mismas variedades mantuvieron el color verde por más tiempo, ya que 12 d después de la cosecha el valor de croma había disminuido en apenas uno o dos puntos y el valor del tono se mantuvo estable alrededor de 130 a 133 (Cuadro 2). Las variedades 'Coloso', '21-20-1' y 'Centauro' registraron un color verde esmeralda, apreciación visual que coincide con el reportado por Ramírez et al. (2007) y correspondió con valores altos de croma (26 a 30) y bajos de tono (124 a 126). Se encontró una correlación negativa entre croma y tono $(r=-0.88, \alpha=0.01)$.

Cuadro 1. Atributos de calidad del fruto de variedades de chile Serrano desarrolladas por Instituto Nacional de Investigaciones Forestales, Agrícolas y Pecuarias y por compañías particulares productoras de semillas.

\begin{tabular}{|c|c|c|c|c|c|c|}
\hline Variedades & $\begin{array}{c}\text { Peso } \\
\text { (g/fruto) }\end{array}$ & $\begin{array}{c}\text { Diámetro } \\
(\mathrm{cm})\end{array}$ & $\begin{array}{c}\text { Longitud } \\
(\mathrm{cm})\end{array}$ & $\begin{array}{l}\text { Firmeza } \\
\left(\mathrm{N} \mathrm{cm}^{-2}\right)\end{array}$ & $\begin{array}{c}\text { Placenta } \\
(\%)\end{array}$ & $\begin{array}{c}\text { Pericarpio } \\
(\mathrm{mm})\end{array}$ \\
\hline 'HS-49' & $10.1 \mathrm{abcd}$ & 1.8 abcd & $7.2 \mathrm{bcd}$ & $147 \mathrm{ab}$ & $100 \mathrm{a}$ & $3.6 \mathrm{a}$ \\
\hline 'HS-51' & $10.5 \mathrm{abcd}$ & $1.9 \mathrm{a}$ & $6.8 \mathrm{~cd}$ & $129 \mathrm{bcd}$ & $99 \mathrm{a}$ & $3.1 \mathrm{a}$ \\
\hline$' 74-27-5$ ' & $9.0 \mathrm{~cd}$ & $1.6 \mathrm{bcd}$ & $7.8 \mathrm{abc}$ & $132 \mathrm{bcd}$ & $97 \mathrm{a}$ & $2.6 \mathrm{a}$ \\
\hline '21-20-1' & $8.3 \mathrm{~cd}$ & $1.8 \mathrm{abcd}$ & $6.2 \mathrm{~d}$ & $147 \mathrm{ab}$ & $100 \mathrm{a}$ & $3.5 \mathrm{a}$ \\
\hline '74-5-5' & $7.8 \mathrm{~cd}$ & $1.6 \mathrm{bcd}$ & $7.4 \mathrm{bcd}$ & $145 \mathrm{ab}$ & $99 \mathrm{a}$ & $3.8 \mathrm{a}$ \\
\hline '33-12-2' & $7.8 \mathrm{~cd}$ & $1.6 \mathrm{bcd}$ & $6.7 \mathrm{~cd}$ & $136 \mathrm{bcd}$ & $99 \mathrm{a}$ & $2.8 \mathrm{a}$ \\
\hline 'Coloso' & $9.2 \mathrm{bcd}$ & $1.7 \mathrm{bcd}$ & $6.7 \mathrm{~cd}$ & 136 bcd & $100 \mathrm{a}$ & $3.1 \mathrm{a}$ \\
\hline 'HS-44' & $7.6 \mathrm{~d}$ & $1.4 \mathrm{~cd}$ & $7.2 \mathrm{bcd}$ & 138 bcd & 99 a & $3.3 \mathrm{a}$ \\
\hline 'Centauro' & $9.7 \mathrm{bcd}$ & $1.6 \mathrm{bcd}$ & $7.0 \mathrm{bcd}$ & $159 \mathrm{a}$ & 99 a & $3.2 \mathrm{a}$ \\
\hline 'Tampiqueño-74' & $8.2 \mathrm{~cd}$ & $1.5 \mathrm{~cd}$ & $7.0 \mathrm{bcd}$ & 127 bcde & $100 \mathrm{a}$ & $3.2 \mathrm{a}$ \\
\hline 'HMX-5651' & $11.8 \mathrm{ab}$ & $1.9 \mathrm{a}$ & $7.1 \mathrm{bcd}$ & $98 \mathrm{e}$ & $100 \mathrm{a}$ & $3.9 \mathrm{a}$ \\
\hline 'HMX-6661' & $11.0 \mathrm{abc}$ & $1.8 \mathrm{abcd}$ & $8.1 \mathrm{abc}$ & $108 \mathrm{de}$ & $100 \mathrm{a}$ & $2.6 \mathrm{a}$ \\
\hline 'HMX-6671' & $10.6 \mathrm{abcd}$ & $1.6 \mathrm{bcd}$ & $8.7 \mathrm{a}$ & $98 \mathrm{e}$ & $100 \mathrm{a}$ & $3.1 \mathrm{a}$ \\
\hline 'Bandido’' & $12.2 \mathrm{a}$ & $1.9 \mathrm{a}$ & $6.8 \mathrm{~cd}$ & $106 \mathrm{de}$ & $98 \mathrm{a}$ & $2.9 \mathrm{a}$ \\
\hline ‘Lucero’' & $11.2 \mathrm{abc}$ & $1.8 \mathrm{abcd}$ & $7.9 \mathrm{abc}$ & $111 \mathrm{de}$ & $96 \mathrm{a}$ & $3.1 \mathrm{a}$ \\
\hline 'Milenio' & $6.9 \mathrm{~d}$ & $1.4 \mathrm{~cd}$ & $6.5 \mathrm{~d}$ & $138 \mathrm{bcd}$ & $100 \mathrm{a}$ & $3.6 \mathrm{a}$ \\
\hline 'Blakie' & 10.5 abcd & $1.7 \mathrm{bcd}$ & 7.5 abcd & 125 bcde & 99 a & $2.6 \mathrm{a}$ \\
\hline ‘Nazas’ & $9.8 \mathrm{bcd}$ & $1.8 \mathrm{abcd}$ & $6.4 \mathrm{~d}$ & 128 bcde & $100 \mathrm{a}$ & $2.5 \mathrm{a}$ \\
\hline 'Tuxtlas' & $10.4 \mathrm{abcd}$ & $1.9 \mathrm{a}$ & $6.5 \mathrm{~d}$ & $139 \mathrm{bcd}$ & 99 a & $3.7 \mathrm{a}$ \\
\hline
\end{tabular}

Medias con letras iguales no son estadísticamente diferentes (Tukey, 0.01).

Cuadro 2. Cambios de color y pérdida fisiológica de peso en frutos de chile Serrano de variedades desarrolladas por el Instituto Nacional de Investigaciones Forestales, Agrícolas y Pecuarias y por compañías particulares productoras de semillas.

\begin{tabular}{|c|c|c|c|c|c|c|}
\hline \multirow{2}{*}{ Variedades } & \multicolumn{2}{|c|}{ Croma } & \multicolumn{2}{|c|}{ Tono } & \multicolumn{2}{|c|}{ PP \% } \\
\hline & $0 \mathrm{DDC}$ & $12 \mathrm{DDC}$ & $0 \mathrm{DDC}$ & $12 \mathrm{DDC}$ & $6 \mathrm{DDC}$ & $12 \mathrm{DDC}$ \\
\hline 'HS-49' & $24 \mathrm{bc}$ & 20 bcde & $127 \mathrm{~d}$ & 127 cde & $11 \mathrm{de}$ & $16 \mathrm{e}$ \\
\hline 'HS-51' & $22 \mathrm{bc}$ & 19 bcde & $128 \mathrm{~d}$ & 128 bcde & $13 \mathrm{de}$ & $19 \mathrm{de}$ \\
\hline '74-27-5' & $21 \mathrm{bc}$ & 18 bcde & $128 \mathrm{~d}$ & 127 cde & $11 \mathrm{de}$ & $18 \mathrm{de}$ \\
\hline '21-20-1' & $27 \mathrm{ab}$ & $24 \mathrm{a}$ & $124 \mathrm{e}$ & $125 \mathrm{e}$ & $13 \mathrm{de}$ & $19 \mathrm{de}$ \\
\hline '74-5-5' & $24 \mathrm{bc}$ & $21 \mathrm{ab}$ & $127 \mathrm{~d}$ & 127 cde & $12 \mathrm{de}$ & $19 \mathrm{de}$ \\
\hline '33-12-2' & $23 \mathrm{bc}$ & 20 bcde & $127 \mathrm{~d}$ & 128 bcde & $12 \mathrm{de}$ & $19 \mathrm{de}$ \\
\hline 'Coloso' & $30 a$ & $24 \mathrm{a}$ & $124 \mathrm{e}$ & $126 \mathrm{de}$ & $16 \mathrm{bc}$ & $26 a b c$ \\
\hline 'HS-44' & $14 \mathrm{~cd}$ & 14 cde & $130 \mathrm{bcd}$ & $130 \mathrm{abc}$ & $9 \mathrm{e}$ & $23 \mathrm{bcd}$ \\
\hline 'Centauro' & $26 a b c$ & $23 \mathrm{ab}$ & $126 \mathrm{~d}$ & 127 cde & $13 \mathrm{de}$ & $23 \mathrm{bcd}$ \\
\hline 'Tampiqueño-74' & $23 \mathrm{bc}$ & $22 \mathrm{ab}$ & $127 \mathrm{~d}$ & $126 \mathrm{de}$ & $14 \mathrm{de}$ & $19 \mathrm{de}$ \\
\hline 'HMX-5651' & $9 \mathrm{~d}$ & $7 \mathrm{e}$ & $134 \mathrm{a}$ & $133 \mathrm{a}$ & $17 \mathrm{bc}$ & $29 a b c$ \\
\hline 'HMX-6661' & $14 \mathrm{~cd}$ & $13 \mathrm{de}$ & $133 \mathrm{ab}$ & $131 \mathrm{ab}$ & $18 \mathrm{bc}$ & $28 \mathrm{abc}$ \\
\hline 'HMX-6671' & $9 \mathrm{~d}$ & $8 \mathrm{e}$ & $133 \mathrm{ab}$ & $132 \mathrm{a}$ & $19 \mathrm{bc}$ & $31 \mathrm{abc}$ \\
\hline 'Bandido' & $10 \mathrm{~d}$ & $9 \mathrm{e}$ & $134 \mathrm{a}$ & $132 \mathrm{a}$ & $19 \mathrm{bc}$ & $30 \mathrm{abc}$ \\
\hline 'Lucero' & $23 \mathrm{bc}$ & 19 bcde & $128 \mathrm{~d}$ & $124 \mathrm{e}$ & $23 \mathrm{a}$ & $34 \mathrm{a}$ \\
\hline 'Milenio' & $25 \mathrm{abc}$ & $22 \mathrm{ab}$ & $127 \mathrm{~d}$ & 127 cde & $13 \mathrm{de}$ & $25 \mathrm{bcd}$ \\
\hline 'Blakie' & $11 \mathrm{~d}$ & $10 \mathrm{e}$ & $132 \mathrm{abc}$ & $131 \mathrm{ab}$ & $19 \mathrm{bc}$ & $30 \mathrm{abc}$ \\
\hline 'Nazas' & $17 \mathrm{~cd}$ & 14 cde & $130 \mathrm{bcd}$ & $130 \mathrm{abc}$ & $18 \mathrm{bc}$ & $27 \mathrm{abc}$ \\
\hline 'Tuxtlas' & $16 \mathrm{~cd}$ & 14 cde & $129 \mathrm{bcd}$ & $130 \mathrm{abc}$ & $21 \mathrm{ab}$ & $35 \mathrm{a}$ \\
\hline
\end{tabular}

PP = pérdida de peso; DDC = días después de cosecha. Medias con letras iguales no son estadísticamente diferentes (Tukey, 0.01). 
Barrera et al. (2005) determinaron valores de tono en C. annuum de $\mathrm{H}^{*}=104^{\circ}$ en estado de madurez fisiológica, con un cambio a $\mathrm{H}^{*}=79^{\circ}$ al llegar al estado de madurez senescente. Según Martínez et al. (2003), el color de fruto de $C$. annuum está influenciado por la fertilización, ya que bajos niveles de potasio resultan en valores bajos de croma y tono, y los bajos niveles de nitrógeno resultan en altos valores de croma y tono.

Durante los $12 \mathrm{~d}$ en que se evaluaron los frutos, las variedades 'HS-49', '74-27-5', '74-5-5', 'Tampiqueño 74', '21-20-1', 'HS-51' y '33-12-2' registraron la menor pérdida de peso (16 a $19 \%$ ), en comparación con el resto de materiales $(P \leq 0.01)$. Por el contrario, las nueve variedades de las compañías particulares presentaron la mayor pérdida de peso ( 25 a $35 \%$ ), en especial las vars. 'Tuxtlas' y 'Lucero' (Cuadro 2). Según Martínez et al. (2005), la pérdida fisiológica de peso en frutos de chile Serrano se explica por efectos genéticos aditivos, y sugieren mejorar esta característica mediante métodos de mejoramiento como la selección genealógica o selección uniseminal. En frutos de $C$. annuum, Díaz-Pérez et al. (2006) encontraron que $26 \%$ de la pérdida de peso se debió a pérdida de agua a través del cáliz.

\section{CONCLUSIONES}

Las variedades 'Bandido' y 'HMX-5651' sobresalieron por su mayor peso e intensidad de color. Las variedades 'HMX-6671', 'HMX-6661', 'Blakie' y 'HS-44' destacaron por su intensidad de color. 'HS-49', 'HS-51', '21-20-1' y '74-5-5' fueron superiores en firmeza y registraron los menores porcentajes de pérdida de peso. 'Centauro' fue la variedad con mayor firmeza. Las variedades '74-27-5', 'Tampiqueño 74' y '33-12-2' presentaron valores bajos de pérdida de peso. Las variedades desarrolladas por las compañías particulares destacaron por su mayor peso e intensidad de color, mientras que las generadas por el INIFAP destacaron por su mayor firmeza y menor pérdida de peso.

\section{BIBLIOGRAFÍA}

Barrera J A, M S Hernández, L M Melgarejo, J P FernándezTrujillo (2005) Physiological changes in amazonic hot pepper accessions during growth, ripening and storage. Acta Hort. 682:302-303.

Díaz-Pérez J C, M D Muy-Rangel, A Gaytán (2006) Fruit size and stage of ripeness affect postharvest water loss in bell pepper fruit (Capsicum annuum L.). HortScience 41:504-505.

Hernández V S, R G López, P Sánchez, M Villarreal, S Parra, F Porras, J Corrales (2008) Variación fenotípica entre y dentro de poblaciones silvestres de chile del noroeste de México. Rev. Fitotec. Mex. 31:323-330.

INIFAP, Instituto Nacional de Investigaciones Forestales, Agrícolas y Pecuarias (1999) Guía Técnica para la Producción Agrícola en el Sur de Tamaulipas. SAGARPA-INIFAP. Publicación Especial. México. 234 p.

Laborde J A, O Pozo (1982) Presente y Pasado del Chile en México. SARH-INIA. Publicación especial No. 85. México. pp:59-60.

Lownds N K, M Banara, P W Bosland (1994) Postharvest water loss and storage quality of nine pepper (Capsicum) cultivars. HortScience 29:191-193.

Martínez Y, L Díaz, J Manzano (2003) Influences of nitrogen and potassium fertilizer on the quality of "Jupiter" pepper (Capsicum annuum) under storage. Acta Hort. 628:30-33.

Martínez Z G, J R A Dorantes, M Ramírez, A de la Rosa, O Pozo (2005) Efectos genéticos y heterosis en la vida de anaquel del chile serrano. Rev. Fitotec. Mex. 28:327-332.

Meneses M I, A Hernández, V G López, A B Vargas, M Ramírez (2006) Características hortícolas de líneas avanzadas de chile jalapeño para el trópico húmedo. Tercera Convención Mundial de Chile. Chihuahua, Chih. 9-11 de julio del 2006. México. pp:61-65.

Pérez-Castañeda L M, G Castañón-Nájera, N Mayek-Pérez (2008) Diversidad morfológica de chiles (Capsicum spp.) en Tabasco, México. Rev. Cuad. Biodiversidad 27:11-22.

Pozo C O (1981) Descripción de Tipos y Cultivares de Chile (Capsicum spp.) en México. Folleto Técnico No. 77. INIA-SARH. México. $40 \mathrm{p}$.

Ramírez M M, G Arcos, H. Mata, E Vázquez (2007) Coloso, Híbrido de Chile Serrano para las Regiones Productoras de México. Folleto técnico No. 21. México. 21 p.

SAS Institute Inc (1998) SAS/STAT User's Guide. Versión 6.01. SAS Institute Inc. Cary, N. Y. USA. 479 p.

Smith L D, J R Stommel, R W M Fung, C Y Wang, B D Whitaker (2006) Influence of cultivar and harvest method on postharvest storage quality of pepper (Capsicum anпиит L.) fruit. Postharv. Biol.Technol. 42:243-247. 